\title{
Análise de Sentimentos em Tweets: um Estudo de Caso sobre os cortes de orçamentos nas IFEs
}

\author{
Danielly Rayanne M. Lima ${ }^{1}$, Raissa Ohana F. O. e Silva ${ }^{1}$, Álvaro Getúlio L. \\ Medeiros $^{1}$, André Luiz F. Alves ${ }^{1}$
}

${ }^{1}$ Instituto Federal de Educação, Ciência e Tecnologia da Paraíba (IFPB) - Picuí, PB Brazil

andre.alvesdifpb.edu.br, [danielly.lima, raissa.ohana, getulio.medeiros] [academico.ifpb.edu.br

Abstract. Understanding the opinions of citizens, especially on the part of governments, is fundamental to decision-making. However, with the large and massive volume of data, process this information manually is a complicated task and does not provide satisfactory results. This research work to build a sentiment classifier enables analyzing opinions automatically using tweets with context of provision cuts made by the brazilian government in the half year of 2019. In order to solve the problem, four machine learning techniques for natural language processing were formulated, where the technique that proved the best result presented an accuracy of $72 \%$.

Resumo. Compreender as opiniões dos cidadãos, principalmente por parte dos governantes, é fundamental para a tomada de decisões. No entanto, com a diversidade de mídias sociais e consequente geração de grande volume de dados, analisar essas informações de forma manual é uma tarefa difícil de ser realizada. Este trabalho tem por objetivo construir um classificador de sentimentos capaz de analisar as opiniões de forma automática envolvendo tweets sobre os cortes orçamentários realizados pelo governo brasileiro no primeiro semestre de 2019. Para isso, quatro técnicas de aprendizagem de máquina para classificação de textos foram comparadas, onde a técnica que obteve o melhor resultado apresentou uma acurácia de $72 \%$.

\section{Introdução}

O crescente uso dos serviços oferecidos na internet e os seus usuários geram uma enorme quantidade de informação. Os usuários contribuem ativamente com o seu conteúdo por meio das aplicações, colaborando assim para a formação de uma inteligência coletiva (O'REILLY, 2007). Essa Web 2.0 proporcionou uma proliferação de informação não estruturada utilizando principalmente as redes sociais, trazendo assim novos desafios e oportunidades na busca e na recuperação da informação (EIRINAKI ET AL., 2012). Compreender o que as pessoas estão pensando ou suas opiniões é fundamental para a tomada de decisões, principalmente no contexto em que as pessoas expressam seus comentários de forma voluntária no intuito de cooperar umas com as outras.

Aplicações de análise de sentimentos no ramo dos negócios buscam analisar os pontos positivos e negativos das empresas ou de seus produtos relatados pelos clientes 
(LIU e SHEN, 2004). Nos trabalhos de (SILVA, 2018) e (AGUIAR, 2013) são discutidas abordagens para análise de sentimentos utilizando métodos léxicos e a técnica Comitê, que combina algoritmos de classificação. Neste trabalho foi feito com os dados de tweets, comparando as métricas de cada modelo separadamente.

No Brasil, com o contingenciamento de recursos financeiros pelo Ministério da Educação no ano de 2019, que afetou todo o sistema federal de ensino, trouxe a tona uma discussão quanto aos investimentos do governo na educação, em especial, elevando os discursos favoráveis ou não ao modelo do ensino gratuito. Nas mídias sociais, esses discursos são propagados através das temáticas abordadas nos movimentos sociais com surgimentos de "hashtags", a exemplo de \#TiraAMaoDoMeuIF, em que evidencia o discurso favorável a investimentos na educação, contrapondo com \#MeuFilhoNaoVai, que defende o contingenciamento de recursos financeiros.

Diante da importância de compreender as opiniões das pessoas, esta pesquisa busca analisar as reações da população referente à atuação das Instituições Federais de Educação (IFEs). O norte da pesquisa é a coleta de textos e análise das opiniões das pessoas nas mídias sociais de forma automática para responder às seguintes problemáticas: é possível construir um classificador de sentimentos capaz de distinguir um texto da mídia social em positivo e negativo? Se sim, como é a distribuição de sentimentos positivos e sentimentos negativos propagados na mídias sociais? As respostas a esses questionamentos possibilitam uma compreensão mais precisa das opiniões dos cidadãos na internet. Neste trabalho, foram comparados os resultados de quatro classificadores de sentimentos que utilizam técnicas de aprendizagem de máquina, onde o classificador que obteve o melhor resultado foi utilizado para inferir os sentimentos de todos os tweets coletados, possibilitando assim a compreensão geral do sentimento expresso pela população na mídia social.

\section{Materiais e Métodos}

A Figura 1 apresenta as principais etapas desenvolvidas neste trabalho. Nesta seção são descritos os materiais e métodos utilizados.

$\begin{array}{llll}\begin{array}{l}\text { Coleta de } \\ \text { dados } \\ \text { (crawler) }\end{array} & \begin{array}{l}\text { Construção } \\ \text { da base de } \\ \text { treinamento } \\ \text { e teste. }\end{array} & \begin{array}{l}\text { Construção } \\ \text { dos } \\ \text { classificadores } \\ \text { de texto }\end{array} & \begin{array}{l}\text { Análise e } \\ \text { sumarização } \\ \text { dos dados. }\end{array}\end{array}$

Figura 1: Etapas da Análise de Sentimentos

O trabalho iniciou com coletas de textos (Coleta de dados) no Twitter $^{l}$, contendo informações correlatas com a temática da educação. Esta busca de textos foi realizada de forma automática através de um crawler (robô de Internet que localiza informações específicas) desenvolvido para coletar as informações textuais por um período de 6 meses, coletando um total de 74.562 tweets. Para desenvolver o crawler foi utilizado a linguagem de programação Python, juntamente com as bibliotecas tweepy e selenium. A Figura 2, apresenta os termos utilizados na coleta de tweets.

\footnotetext{
${ }^{1}$ www.twitter.com
} 


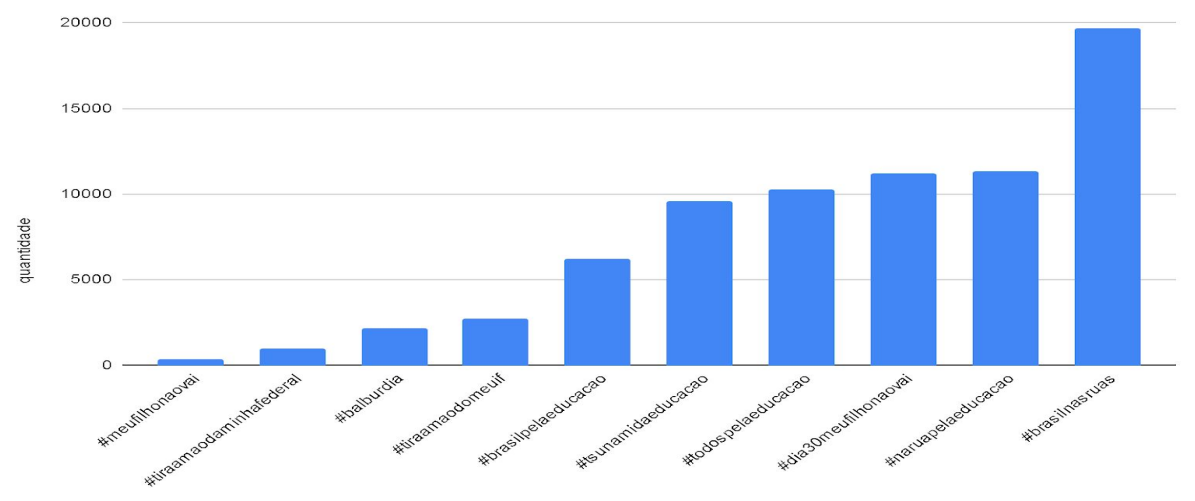

Figura 2: Quantidade de tweets por termo de busca

Dos textos coletados, foram separados 5.000 deles, aleatoriamente, para compor a base de treinamento e teste dos modelos de classificação. Três pessoas rotularam o sentimento dos textos nos sentimentos positivo, negativo ou neutro Para saber o sentimento do texto predominante foi utilizado o sistema de voto majoritário. $\mathrm{O}$ processo de rotulação do sentimento resultou em 1.648 tweets positivos, 587 negativos e 2.765 neutros. Além do mais, para obter melhores resultados, foi necessário aplicar técnicas de pré-processamento em todos os textos coletados. Essa etapa consiste na limpeza dos dados, como a remoção das stopwords, dos caracteres especiais, links e imagens, além de conversão de todas as palavras para minúsculo.

O modelos de classificação utilizam abordagem de aprendizagem de máquina e foram treinados a partir da base de treinamento. Para a construir o classificador de texto foi escolhida a linguagem de programação Python. Neste trabalho foram implementados quatro técnicas de classificação, a saber: Naive-Bayes, Árvore de Decisão, Máquina de Vetores de Suporte (SVM) e Rede Neural. Após comparar os resultados, todos os tweets coletados foram classificados, possibilitando a compreensão das opiniões da população, através da análise e sumarização das opiniões.

\section{Resultados}

Os classificadores foram avaliados considerando $70 \%$ e $30 \%$ de dados para treinamento e teste, respectivamente. A Tabela 1 apresenta as métricas de avaliação dos modelos. Observa-se que o modelo SVM apresentou o melhor resultado.

Tabela 1: Resultado comparativo do classificadores

\begin{tabular}{|c|c|c|c|c|}
\hline Modelo & Árvore de Decisão & Naive-Bayes & SVM & Redes Neurais \\
\hline Acurácia & $61 \%$ & $64 \%$ & $72 \%$ & $71 \%$ \\
\hline Precision & $62 \%$ & $63 \%$ & $70 \%$ & $71 \%$ \\
\hline Recall & $61 \%$ & $64 \%$ & $72 \%$ & $71 \%$ \\
\hline f1_score & $61 \%$ & $64 \%$ & $71 \%$ & $70 \%$ \\
\hline
\end{tabular}

Uma vez treinado o classificador de sentimentos, todos os 74.562 tweets foram rotulados utilizando o modelo. O gráfico da Figura 3 apresenta as quantidades de sentimentos positivos e negativos, por dia, considerando o período entre o início de 
maio e o ínicio de julho, que teve maior expressão de sentimentos. Em alguns momentos a quantidade de sentimentos positivos foi bem superior aos negativos.

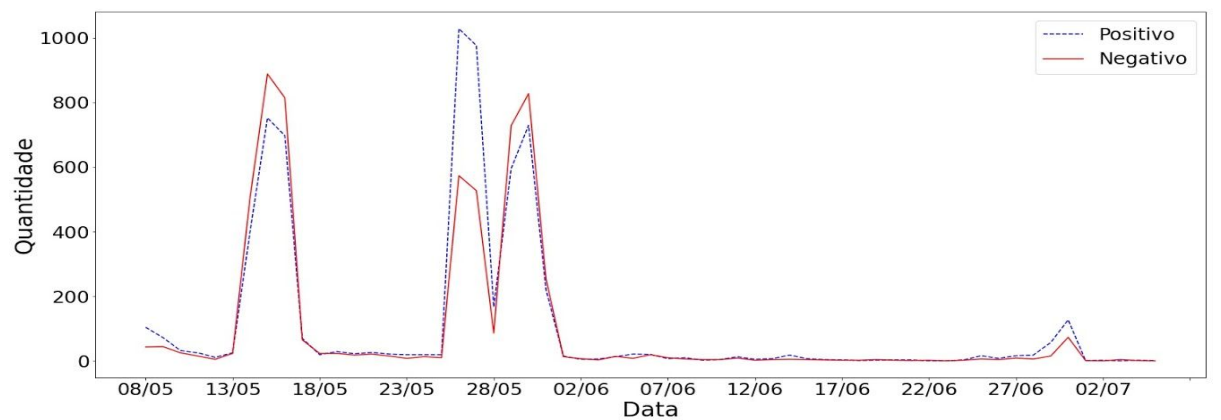

Figura 3: Sumarização das opiniões por dia

\section{Considerações Finais}

O objetivo da análise de sentimentos é extrair a opinião e o conhecimento subjetivo de textos, formalizar esse conhecimento descoberto e analisá-lo para uso específico. $\mathrm{O}$ estudo em questão coletou tweets com a temática da educação no país, analisando de forma automática as opiniões dos usuários. Foram coletados tweets relacionados à educação e construído classificadores de textos para a inferência de opiniões contidas nos tweets. Comparando o resultados das técnicas de classificação Árvore de Decisão, Naive-Bayes, SVM e Redes de Neurais, observou-e que estas duas últimas obtiveram os melhores resultados, apresentando acurácias de $72 \%$ e $71 \%$, respectivamente. Com esta pesquisa, espera-se possibilitar uma análise automática dos textos em mídias sociais para a compreensão dos sentimentos da sociedade relativos à educação no país. Como trabalhos futuros, pretende-se melhorar o conjunto de treinamento, inclusive com o balanceamento das quantidades de cada classe, estudar outros tipos de redes neurais (Deep Learning), técnicas de sumarização das opiniões, bem como analisar as interações em cada tweet, considerando curtidas e respostas para uma melhor análise.

\section{References}

EIRINAKI, Magdalini, PISAL, Shamit, SINGH, Japinder (2012). Feature-based opinion mining and ranking. Journal of Computer and System Sciences, Vol 78, p. 1175-1184.

LIU, N., \& Shen, B. (2020). ReMemNN: A novel memory neural network for powerful interaction in aspect-based sentiment analysis. Neurocomputing.

O'REILLY, Tim (2007). What Is Web 2.0: Design Patterns and Business Models for the Next Generation of Software. Communications \& Strategies, Vol 1, p. 17-37.

SILVA, N. F. F. Um estudo comparativo sobre métodos de análise de sentimentos em tweets. Revista de Sistemas de Informação da FSMA, v. 1, n. 22, p. 35-48, 2018.

AGUIAR, Erikson Júlio et al. Análise de sentimento em redes sociais para a língua portuguesa utilizando algoritmos de classificação. In: Anais do XXXVI Simpósio Brasileiro de Redes de Computadores e Sistemas Distribuídos. SBC, 2018. 